ist in dieser Hinsicht durch alte Überarbeitungen mit dogmatischer Tendenz besonders interessant. Es genügt, dafür auf den kritischen Apparat zu verweisen.

Indessen ist hier noch an einige weitere Überarbeitungen zu erinnern, die einer andern Stelle entnommen sind, weil sie mir in ähnlicher Weise durch antinestorianische Besorgnisse motiviert scheinen. Sie finden sich gleichfalls in ein Glaubensbekenntnis eingeschoben 224, 1: ả $\pi 0 \delta \iota \delta o ́ v \tau \alpha$

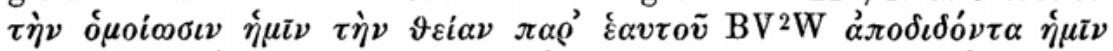

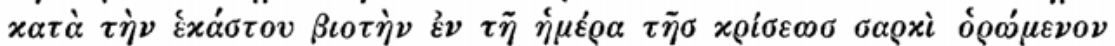

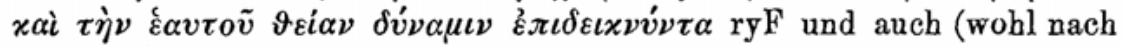
einer Randvariante) W.

Um das Motiv zur Änderung zu verstehen, sind zu vergleichen der IX.u. XI. Anathematismus des Cyrill nebst den Gegenanathematismen des Nestorius, außerdem das Glaubensbekenntnis des Theodorus von Mopsuestia (Hahn, Bibliothek der Symbole ${ }^{3}$, S. 304, 9-11). Auf der folgenden

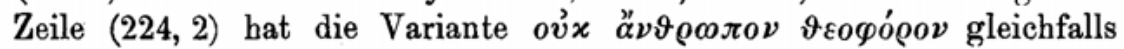
den Zweck, eine nestorianische Auslegung zu verhindern, an welche die Verfasser der Urkunde noch nicht dachten.

Natärlich haben die Änderungen nicht immer eine so specielle Tendenz, sondern sie sollen oft einfach Sinn in eine schwierige oder verderbte Stelle bringen. In dieser Hinsicht genügt es, auf den kritischen Apparat zu verweisen. Dort liegt offen zutage, was die Besserungen z. B. aus dem Symbol von Sardica II 8 gemacht haben.

Man beachte auch die kritische Bemerkung zu 255, 7, wo ich eine

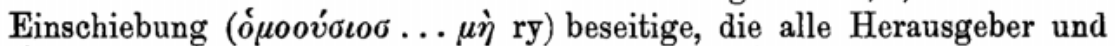
Übersetzer getäuscht hatte.

Die Textgeschichte Theodorets zeigt die im allgemeinen so häufige Tendenz, in alle Handschriften mehr und mehr die lectio facilior oder einfach die Correcturen einer neuen Recension einzuführen. Der Fall des Codex A, wo $\mathrm{A}^{\mathrm{c}}$ überall die richtigen Lesarten von $\mathrm{A}$ unterdrückt, ist dafür das charakteristischste Beispiel.

\title{
VI. Die Handschrift der Tripartita
}

In der Tripartita des Theodorus Lector haben wir das Glück für viele Stellen Theodorets einen Text zu besitzen, dessen Überlieferung sich von der unsrer Handschriften seit dem sechsten Jahrhundert losgelöst hat. Nur stammt der Marcianus, die einzige Handschrift, in der die Tripartita erhalten ist, aus dem XIII. Jahrhundert, und als erste Frage drängt sich die auf, bis zu welchem Grade der von Theodorus abgeschriebene Text während jener sieben Jahrhunderte 
Entstellungen erfahren hat. Glücklicherweise sind wir in dieser Hinsicht nicht ohne Kontrollmittel. Bidez (La tradition manuscrite de Sozomène et la Tripartite de Théodore le Lecteur. TU XXXII $2^{\text {b}}$, S. $51 \mathrm{ff}$ ) hat gezeigt, daß Cassiodor bis zum Cap. 7 des Buches II den Text der Tripartita sklavisch übersetzt hat, während er von da an ganz unabhängig vorgeht.

Es ist demnach für den Abschnitt gemeinsamen Ursprungs lehrreich, die Texte des Theodoret in der Tripartita und den des Cassiodor in Bezug auf ihren Zustand zu vergleichen. Man stößt dabei zuerst auf Febler, die $\mathrm{T}$ und Cass. gemeinsam sind, ein Beweis, daß schon zur Zeit des Cassiodor die Handschrift in keinem vollkommenen Zustand war. Aber die Vergleichung offenbart auch, daß zwischen jener Zeit und der der Handschrift der Marciana die Überlieferung der Tripartita zahlreiche weitere Entstellungen erlitten hat.

Der Codex T hat nicht nur Auslassungen und neue Fehler - das würde das geringste Übel sein -, sondern er weist auch Änderungen auf, denen der Charakter der Willkblirlichkeit nicht abgesprochen werden kann.

Auslassungen in TCass.: 7, 3. 9, 23. 10, 2. 15, 26. 35, 10. 36, 10/11. Andre Fehler: 11, 12 u. 22. 13, 2 u. 10. 24, 17. 27, 1 usw.

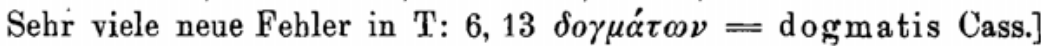
$\delta \iota \delta \alpha \gamma \mu \alpha \dot{\tau} \tau \omega \nu$ T; vgl. auf derselben Seite Z. 18, 20, 21 u. 23. - 7, 10. 8, $6,15 / 16$ u. 17. $9,3.19,11.29,1.35,23$.

Neue Auslassungen: 15, 16 u. 25. 21, 7. 26, 6.

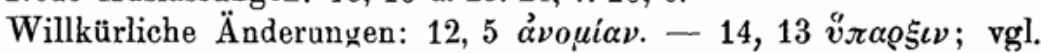
12, 10/11. 15, 27. 18, 5. 20, 2.

Von der Stelle ab, wo T und Cassiodor auseinandergehen (S. 37), stellt Cass. immer eine bessere Handschrift dar, als es der Marcianus T gegenwärtig ist.

Bei den Entstellungen der Überlieferung Theodorets in T ist jedoch in Rechnung zu ziehen, daß manche davon sicher auf Theodorus selber zurückgehen. Beispiele dafür finden sich in dem Teile von $\mathrm{T}$, dessen Alter durch die Übersetzung Cassiodors bestätigt wird; unter anderm biblische Zusätze: 12,18. 28, 21. Bei ähnlichen Einschiebungen in den Abschnitten, wo Cass. von $\mathrm{T}$ unabbängig ist, dürfte die Vermutung zulässig sein, daß auch sie auf den Verfasser der Tripartita zurückgehen, z. B. 115, 2. 151, 2.

Ferner verfährt Theodorus an den Stellen, wo er seine verschiedenen Quellen zusammenbringt, ziemlich frei, und es kommt vor, daß er sie umgestaltet und durcheinanderwirft (vgl. Bidez a.a.0. $52 \mathrm{ff}$ ). Selbst im Verlauf eines Capitels, das er ganz und gar einem Autor entnimmt, fügt er zuweilen ein anderswoher entlehntes Detail hinzu und macht seinen Text demgemäß zurecht. Ein von der Übersetzung 
des Cass. bestätigtes Beispiel findet sich 32, 9/10. Theodorus hat (im Anschluß an Sozomenus I 19) dem Eusebius die Rolle zurtickgeben wollen, die Theodoret dem Eustathius in der Sitzung des Concils zu Nicäa vorbehalten hatte.

Äbnlich ist $34,1-3$ die Auslassung in $\mathrm{T}=$ Cass. eine beabsichtigte; s. krit. App.

Analoge Überarbeitungen, die wahrscheinlich auf Theodorus zurückgehen, finden sich im ganzen Verlauf der Tripartita, und um den Leser darauf hinzuweisen, daß das Zeugnis des Codex $\mathrm{T}$ in diesem Fall kein Gewicht mehr hat, habe ich ihn im kritischen Apparat mit $\mathrm{T}^{*}$ bezeichnet. Zahlreiche Beispiele solcher Änderungen siehe S. 70-72. 97, 11/12. 119,1 ff. $162,16.170,13$ ff. 173, 1 usw.

$\mathrm{T}$ hat übrigens von allen unsern Handschriften am wenigsten Bedenken, tendenziöse Einschiebungen vorzunehmen, selbst in den Urkunden. Zum Beispiel interpoliert II 8 ohne Zweifel Theodorus selber dreimal den Text, um Paulus von Constantinopel auf dem Concil von Sardica in die Gesellschaft des Athanasius, Marcellus und Asclepas zu bringen: 103, 14. 106, 3. 110, 17. - Vgl. auch 112, 3 u. 18. 144, 23.

Der Ausdruck $v \pi \dot{o} \sigma \tau \alpha \sigma \iota s$ in derselben Urkunde II 8 war offenbar der Theologie des Theodorus anstößig. Er hat ihn grundsätzlich und ausnahmslos durch ovoía ersetzt: 113,9 u. 13. 115, 11. 116, 4 u. 16. Ebenso in einer andern Urkunde 148, 9.

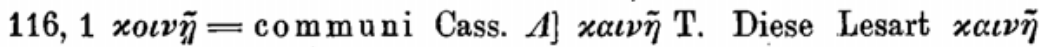
in T würde sich, selbst wenn sie, wie Loofs glaubt, die richtige ist, für mich am wahrscheinlichsten durch eine Änderung oder durch ein glückliches Verschreiben erklären.

Die S. XXVIII und XXXII angeführten feblerbaften Übereinstimmungen von $\mathbf{T}$ mit der Quelle von A und $\mathrm{n}$ bilden ein Mittel, das Alter einiger Lesarten von $\mathrm{T}$ auf anderm Wege nachzuweisen.

Ferner lassen auch Fälle merkwürdigen Zusammentreffens zwischen T und dem Text des Gelasius Cyzicenus, wo dieser Theodoret abschreibt, Schlüsse zu. Solche speciellen Übereinstimmungen finden sich besonders 43, 3. 46, 3. 61, 8. 78, 3. 82, 7. 83, 18. Diese Anzeichen, deren Bedeutsamkeit die Prüfung des kritischen Apparats dartut, scheinen mir den Schluß zu rechtfertigen, daß sich Gelasius und Theodorus Lector zweier Handschriften des Theodoret bedient haben, die zu derselben Recension gehörten.

Endlich lassen sich wohl als auf die ursprüngliche Tripartita zurückgehend manche alten Fehler auffassen, in denen T mit guten Handschriften übereinstimmt. In dieser Hinsicht ist $T$ lehrreich, um zu zeigen, wie früh die Varianten und Überarbeitungen in den Text ein- 
gedrungen sind; siehe z. B. 22, 19. 103, 19/20. 104, 15. 108, 3. 111, 14 . 112, 19. 117,1 . 119,22 . 135,8 u. 18. 141,9 . $148,3 / 4$.

Eine Überarbeitung, die den alten Text in $\mathbf{v}$ verdrängt hat, ist in gleicher Weise neben der richtigen Lesart in T 164, 20/21 eingedrungen und dadurch als sehr alt erwiesen.

Außer den alten Fehlern und Überarbeitungen findet sich in $\mathrm{T}$ noch eine Fülle specieller Lesarten, die sich am wahrscheinlichsten so erklären, daß sie im Lauf der besondern Überlieferung dieser Handschrift entstanden sind. Sie begegnen auf jeder Seite des kritischen Apparats, und es ist überflüssig, hier Beispiele anzuführen.

Ich habe hier etwas den Stab über den Codex T gebrochen, aber um ihn gerecht zu beurteilen, darf man die zahlreichen Fälle nicht außer Ansatz lassen, wo er sich den besten Handschriften an die Seite stellt und uns so große Dienste leistet.

\section{Cassiodor}

Der außerordentliche Wert, den die Übersetzung des Cassiodor ${ }^{1}$ ) für die Arbeit der Auswahl unter den Lesarten besitzt, ist schon oben hervorgetreten. Cassiodor darf mit B, dem seine Quelle nahe stand (s. S. XXXVIf), als einer der treusten Vertreter der Überlieferung betrachtet werden.

Ich habe bereits (S. XLIX) festgestellt, daß da, wo er Theodorus selber übersetzt, sein Text im allgemeinen von den Entstellungen unsrer Handschrift der Tripartita frei geblieben ist. Sobald er seiner eignen Handschrift von Theodoret folgt, stellt er immer eine bessere Überlieferung als T dar. Es genügt z. B. ein Blick auf die ganze lange Urkunde II 8, um zu sehen, daß die Überlieferung Cassiodors vortrefflich ist, und die Kontrolle seiner Handschriften hat mir zuweilen ermöglicht, sie noch zu verbessern; z. B. 114, 5. Da boten mir die Ausgaben von Cass. omnibus $=\pi \tilde{\alpha} \sigma \iota \nu$, Fehler von $\mathrm{T}$. Aber in seinen besten Handschriften (LP) fand ich omnia omnibus $=\pi \alpha \nu \tau \alpha \dot{\alpha} \alpha \sigma \iota \nu$ Brv, wodurch die peinliche Treue des Übersetzers bestätigt wird. Ich habe den Eindruck, daß, gerade umgekehrt wie Theodorus Lector, Cassiodor niemals, wenigstens soweit es Theodoret betrifft, den Text seiner griechischen Handschrift mit Vorbedacht umgearbeitet oder gefälscht hat; s. in dieser Hinsicht seine mit B und $V^{2}$ gemeinsamen Fehler (S. XXXVII u. XL).

1) Ich habe durchweg den Namen Cassiodor und nicht Epiphanius gebraucht, unter anderm deshalb, weil der letztere Name (s. S. $25 \mathrm{ff}$ ) für einen andern Epiphanius aufgespart werden sollte. 